\title{
Trends of Malaria on Hotspot and Ivermectin Mass- drug Administration Zone of Amhara Regional State, 2020
}

Chalachew Yenew ( $\nabla$ chalachewyenew50@gmail.com )

Debre Tabor University

\section{Sileshi Mulatu}

Bahir Dar University

\section{Research Article}

Keywords: Malaria Trend, Ivermectin, Mass-drug Administration, Ethiopia

Posted Date: December 16th, 2020

DOI: https://doi.org/10.21203/rs.3.rs-112470/v1

License: (c) (i) This work is licensed under a Creative Commons Attribution 4.0 International License.

Read Full License 


\section{Trends of Malaria on Hotspot and Ivermectin Mass-drug Administration Zone of Amhara Regional State, 2020}

Chalachew Yenew ${ }^{* 1}$, Sileshi Mulatu ${ }^{2}$

${ }^{1}$ Social and Population Health (SPH) Unit, Debre Tabor University, Ethiopia

${ }^{2}$ Bahir Dar University, Ethiopia

Correspondence: Chalachew Yenew

Email: Chalachewyenew50@gmail.com

Phone number: +251945563008

Social and Population Health (SPH) Unit, Debre Tabor University, Ethiopia 


\section{ABSTRACT}

Background:- Public health surveillance (PHS) is the continuing organized gathering, investigation, elucidation, and well-timed distribution of health-related information for activities and program evaluation. Conducting a surveillance system evaluation is crucial for monitoring the efficacy and effectiveness of intervention programs in health care systems. This study aimed to Evaluate the Trends of Malaria in the hotspot and Ivermectin mass-drug administration Zone of Amhara Regional State, Ethiopia, 2020.

Methods: - A descriptive prevalence study design was used to evaluate the surveillance system of the Awi zone selected woreda. 25 study sources were incorporated in the survey (5 District Health Offices (5HOs), 10 Health Centers (10HCs), and 10 Health Posts (10 HPs). Purposive sampling techniques were utilized. Data were obtained by communicable diseases control the standard format of systematic evaluation of four surveillance units from January to August 2020 through observation, document review, and interviewing surveillance officers and focal persons using a semi-structured Survey.

Results: - Average report fullness and aptness were $97.9 \%$ and $96 \%$ respectively. The average annual malaria incidence rate was a decline from the year 2015 to 2019 with an average reduction rate of $5.5 \%$ and the average annual parasitic incidence rate was 52 (22-199). In 2019/2020, 43131 Malaria cases were reported in the zone. Supervisions were made as integrated supportive supervision in the last six months. However, there was no programspecific supportive supervision of public health emergency management. Data analysis was not routinely practiced in both visited districts and was not used for decision making.

Conclusions: This result revealed that the malaria incidence rate showed a remarkable decline. However, the annual parasitic incidence rate remains constant. This indicates that ivermectin did not affect malaria elimination. The structure of the surveillance information transfer as of Kebel to Zone was well organized. However, coordination and supervision of the surveillance activities were not frequent. From those supervised health facilities, most of them are not receiving feedback. There was no budget line, written feedback, epidemic and preparedness, and a response plan regular based on supportive supervision at all visited health facilities. Depending on this, we recommend that districts and sub-city health offices should conduct regular surveillance data analysis, perform supportive supervision, avail budgets and mitigate resource constraints and improve data quality on the job training and supportive supervision. Further laboratory investigations should be done to investigate the effect of ivermectin on the parasites under laboratory conditions.

Keywords: Malaria Trend, Ivermectin, Mass-drug Administration, Ethiopia 


\section{Background}

Intestinal parasitic diseases like malaria are curable. But, life-threatening infections cause intense febrile sickness, which taints the human liver and red blood cells. It is transmitted from individual to individual by the chomp of the Anopheles mosquito, which chomps as it were at night. It is transmitted to individuals via the chomps of Anopheles mosquitoes, called malaria vectors, taints with Plasmodium parasites (PPs) (1).

In 2018, assessed 228 million diseases of malaria occurred globally (95\% confidence interval (CI): 206-258 million), in contrast with 251 million diseases in 2010 (95\% confidence interval (CI): 231-278 million) and 231 million infections in 2017 (95\% confidence interval (CI): 211259 million). Most malaria diseases in 2018 were within World Health Organization (WHO) report, African Region (213 million or 93\%), followed by the South-East Asia Region with $3.4 \%$ of the diseases and the Eastern Mediterranean Region with $2.1 \%$ and In 2018, there were an estimated 405000 mortality from malaria globally, compared with 416000 determining mortality in 2017, and 585000 in 2010 (2).

Public health surveillance (PHS) is the continuing organized gathering, investigation, elucidation, and well-timed distribution of health-related information for activities and program evaluation. Sustain the decrease of the trouble of immediately reportable diseases (IRDs), eradicate the disease, and prevent its restitution is the main aim (3).

According to Ethiopia, malaria elimination strategy, mortality, and morbidity attributed to malaria declined significantly during the review period. Accordingly, death due to malaria has declined by $67 \%$ from $0.9 / 100,000$ population to $0.3 / 100,000$ population at risk between 2016 and 2019. Similarly, the annual parasite incidence (API) has declined by $37 \%$ from the $19 / 1000$ population to $12 / 1000$ population between 2016 and 2019. The number of confirmed malaria cases has reduced by $47 \%$ between 2016 and 2019 (4).

PHS of infectious diseases is recognized as the keystone of Public Health (PH) decision-making and practice. PHS data are crucial for monitoring the health status of the population, detecting diseases, and triggering actions to prevent further illness and ensure that these diseases are monitored efficiently and effectively (5).

WHO recommends mass drug administration (MDA) of ivermectin as a potential tool to reduce malaria transmission to reach malaria vectors that feed on the temporal and spatial gaps left by core vector control interventions (long-lasting insecticidal nets and indoor residual spraying (6). Information distributed by a PHS system can be used for immediate $\mathrm{PH}$ action, program planning and evaluation, and formulating research suggestions. The PHS system has been 
developed to address a range of $\mathrm{PH}$ needs. They include a variety of data sources essential to $\mathrm{PH}$ action (7)

Malaria PHS predicts the maintenance of continuing watch over the status of malaria in a community. The main purpose of PHS is to detect changes in trends or distribution of malaria and other vector-borne diseases to initiate investigation or control measures. The aim of malaria PHS in the prevention and control of malaria in the community (8).

Conducting PHS is key for monitoring the efficacy and effectiveness of interventional programs in the health care system. Effective PHS systems are one of the basic strategies of national disease prevention and control programs. A PHS scheme serves 2 relevant roles; (i) untimely caution of likely threats to $\mathrm{PH}$ and (ii) program monitoring of the PHE, which could be emerging-definite or multiple-emergency in life (9). Therefore, the purpose of this study was to evaluate Trends of Malaria in the hotspot and Ivermectin mass-drug administration Zone of Amhara Regional State, Ethiopia, 2020. 


\section{Methods}

\section{Study Design and Period}

The institution-based prevalence study design was used based on an "overview of evaluating PHS systems CDCs updated in the 2012 guideline for evaluating PHS system" as a framework for the evaluation to achieve the stated objective of the study from June 2020 to August 2020.

\section{Study unit}

The study units were woreda HOs and health facilities (HF). 25 study sources were included in the survey, including woreda $\mathrm{HOs}=5, \mathrm{HCs}=10$, and $\mathrm{HPs}=10$.

\section{Sample size and techniques}

A Zone is one of the Zone in the Amhara Region that reported an uppermost figure of cases in the region during the study. We purposely selected to conduct the PHS system evaluation in this zone. 25 sites were selected for the study. About 5(50\%) woredas are selected by a convenient sampling method based on their malaria weekly report caseload and surveillance performances. For each selected Woredas, in each selected HO: $10 \mathrm{HC}$ and each selected HC: $10 \mathrm{HP}$ was selected by purposive.

\section{Selection of health facilities}

HFs were selected by discussing with district PHEM officers, two HCs and two HP from each woreda were included in the evaluation, similarly, HC was included based on the 2019/2020 PHEM performance by taking HCs that had better performance and least performance. HPs were selected by purposive.

\section{Data collection and Data source}

Data collection would be done by face to face interviews using questionnaires/checklists. Answers from respondents (HO head and/or PHEM officers) and observation of tools for surveillance and secondary data review. And the increasing or decreasing rate before and after ivermectin mass drug administration was measured by the annual parasitic incidence rate. And the population data used to determine the annual parasitic incidence rate and disease rate.

\section{Data Management and Analysis}

The data were coded and entered using Epi info 7 and exported to the Statistical Package for the Social Sciences (SPSS) version 20. Then the mean prevalence, variability, and linear regression were executed by using SPSS statistical software version 20. The variances between 
groups were handled by analysis of variance (ANOVA). Results are presented with text descriptions, graphs, and tables.

\section{Data Quality assurance}

Data was reviewed by PH experts who have worked in the PHS system. Some data was deduplicated, especially the data from malaria monitoring charts, weekly PHEM reports, and year reports. We cleaned and analyzed data using Microsoft Office Excel 2016 and Epi-data to show report completeness, timeliness, and trends over time and the geographical distributions. 


\section{RESULTS}

The average completeness of the weekly report collected from $2015-2019$ was $97.9 \%$ (95\% CI: 96.2\%, 98.9\%) and the average report timeliness was $96 \%$ (95\% CI: 95.5\%, 97.7\%) (Fig. 1).

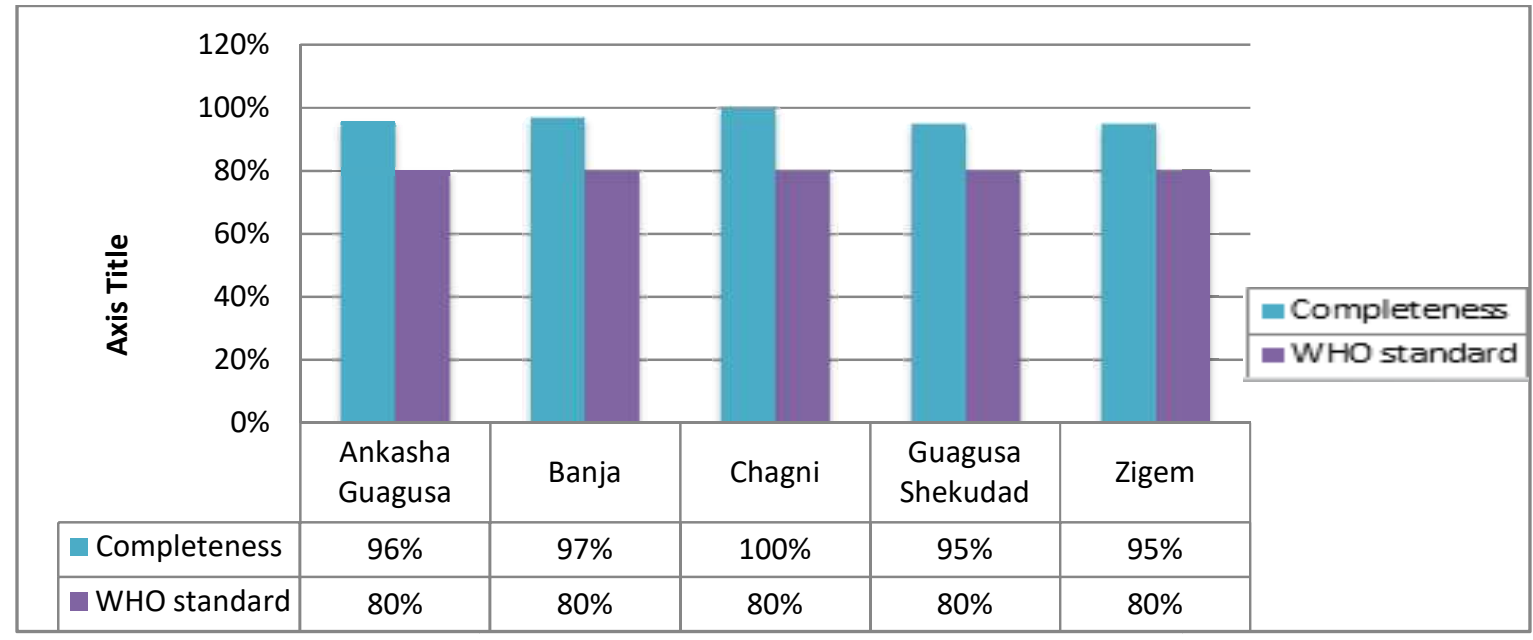

Figure 1: Timelines \& completeness of Governmental Health Facility Awi Zone, Amhara Regional State, Ethiopia, 2020.

The overall report completeness and timeliness rate was above the World Health Organization minimum goals (80\%) in the last five years (Fig. 1). This might be due to increasing awareness of the community and acceptance of the community, health extension workers, and other health providers on of the PHS.

In this analysis, we were done a malaria incidence rate of the zone and woreda according to the previous 5-year data to identify the hotspot zone and woreda. Our current weekly collected data from the year 2015-2019 provided that, North Gondar (59.7), Awi (54.3), South Gondar (27.5), and West Gojam (26.3) per 1000 populations had relatively the highest annual average incidence rate (Fig.2).

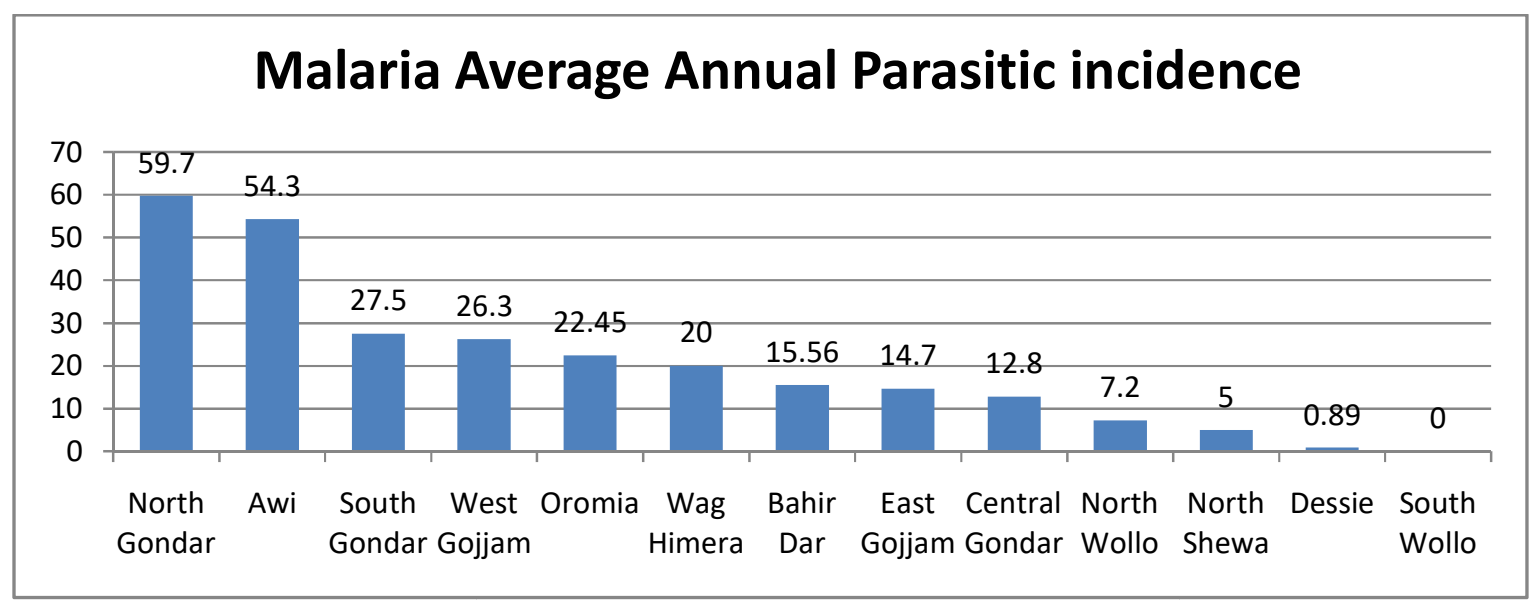

Figure 2: Malaria average annual parasitic incidence rate per 1000 population by zones in Amhara Region, Ethiopia, 2015-2019. 
In the current study, the Awi zone is our study area because the second hotspot area and all woreda were Ivermectin mass drug administration targeted woreda from the Year 2015-2019 and malaria incidence rate (Table 1 and Fig. 3).

Table 1: Malaria incidence rate of the study area from 2015-2019, Awi zone, Amhara Region

\begin{tabular}{|l|l|l|l|l|l|l|}
\hline s.no & Woreda & 2015 & 2016 & 2017 & 2018 & 2019 \\
\hline 1 & Ankesha & 35.70 & 22.70 & 11.33 & 7.30 & 7.26 \\
\hline 2 & Banja & 56.85 & 24.80 & 15.75 & 10.21 & 12.46 \\
\hline 3 & Chagni & 160.20 & 88.56 & 53.22 & 59.57 & 80.91 \\
\hline 4 & Dangila Ketama & 21.97 & 10.52 & 9.37 & 7.66 & 9.71 \\
\hline 5 & Dangila zuria & 28.06 & 16.42 & 12.41 & 8.18 & 13.85 \\
\hline 6 & Fagita lokoma & 30.01 & 14.88 & 9.67 & 8.81 & 4.82 \\
\hline 7 & Guagusa shekudade & 47.74 & 18.30 & 13.37 & 7.96 & 6.23 \\
\hline 8 & Guanga & 44.51 & 31.48 & 13.74 & 8.57 & 22.82 \\
\hline 9 & Injebra & 45.76 & 15.06 & 12.88 & 8.18 & 8.98 \\
10 & Jawi & 333.24 & 237.06 & 154.71 & 310.84 & 175.48 \\
\hline 11 & Zigem & 50.82 & 28.34 & 26.03 & 41.40 & 28.20 \\
\hline
\end{tabular}

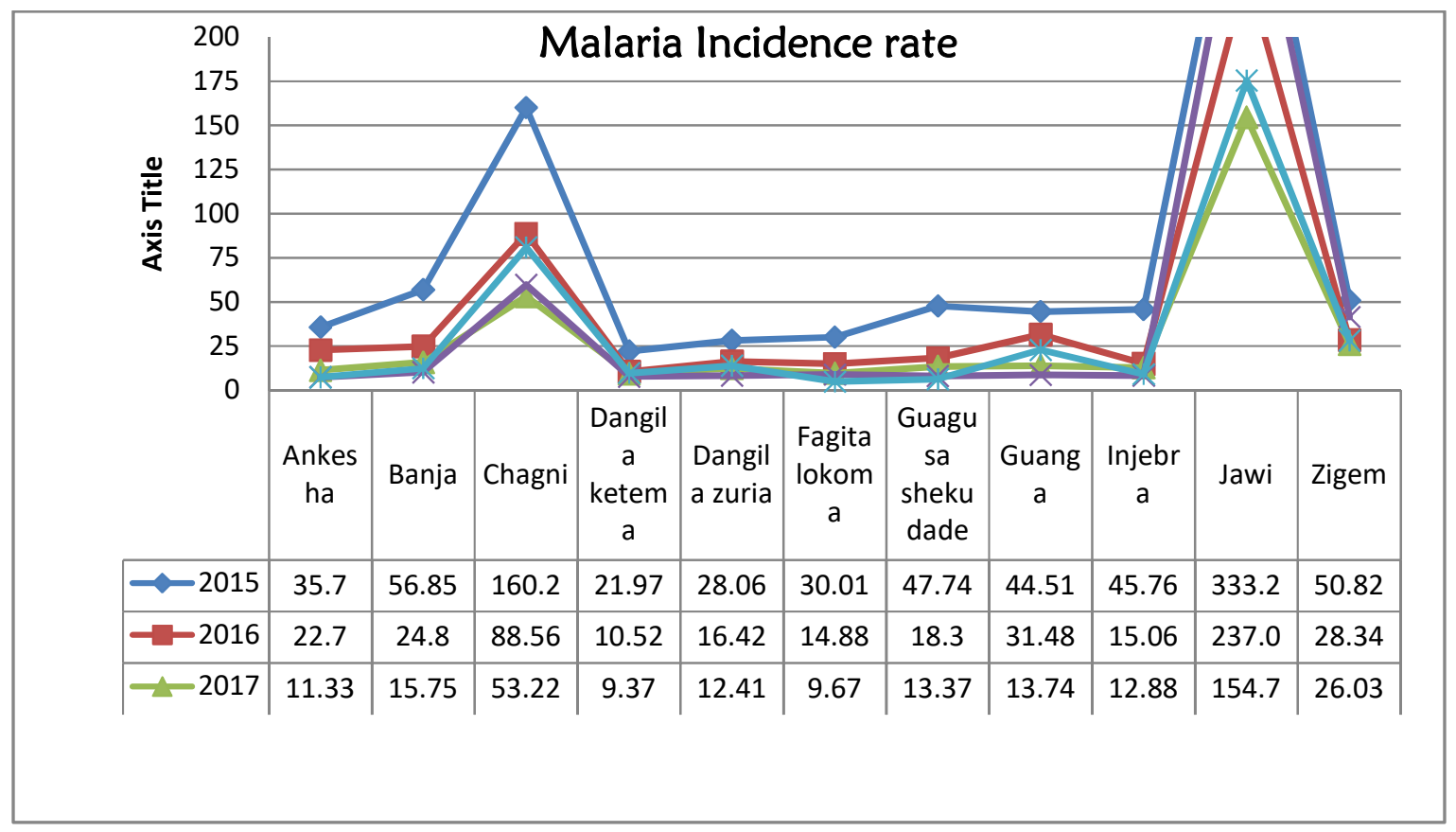

Figure 3: Trends of Malaria Incidence rate by Years of Awi Zone Amhara Region, 2020. 


\section{Annual parasitic incidence (API) rate}

The main indicator of the effect of Ivermectin on malaria elimination here in the Figure below indicates that the current API rate. The woreda with its malaria incidence rate reported from 2015-2019 was present in Figure 4.

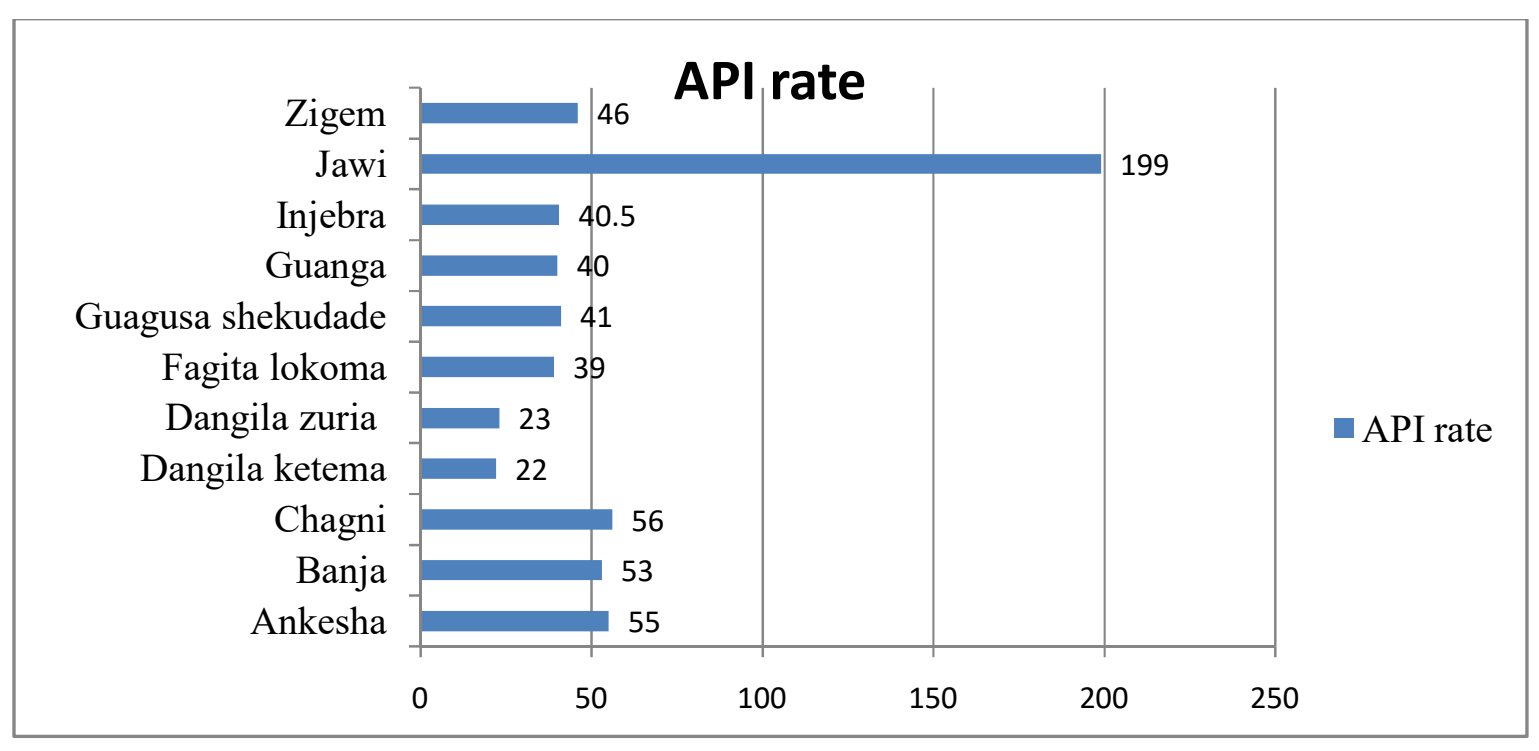

Figure 4: API rate of the study area, Awi Zone, Amhara Region, 2019.

The current finding indicates that Ivermectin did not affect the parasitic incidence rate reduction. It implies that Ivermectin was not effective in malaria elimination in Awi Zone, Amhara Regional, 2020.

\section{Population under PHS}

PHEM targets all the population to be under surveillance for all the 21 diseases nationally and 22 diseases (include Lehimaniasis) in the Amhara region. A population under PHS includes all residents selected Woreda of Awi Zone and Visited Woredas by 2020 ( Table 2).

Table 2:-Population under Surveillance of Awi Zone and Visited Woredas 2020

\begin{tabular}{|l|l|l|l|l|}
\hline S.No & Zone/Woreda Name & Total Population & Male & Female \\
\hline 1 & Chagni & 48,260 & 24,613 & 23,647 \\
\hline 2 & Ankasha Guagusa & 108,376 & 55,272 & 53,104 \\
\hline 3 & Banja & 99,967 & 50,983 & 48,984 \\
\hline 4 & Zigem & 109,255 & 55,720 & 53,535 \\
\hline 5 & Guagusa Shekudad & 108,154 & 55,159 & 52,995 \\
& Total & $\mathbf{4 7 4 , 0 1 2}$ & $\mathbf{2 4 1 , 7 4 6}$ & $\mathbf{2 3 2 , 2 6 6}$ \\
\hline
\end{tabular}

The national PHEM targets all the population in the country to be under PHS for all the 22 priority diseases. The Awi zone cascade the same structure, with a total population of $\mathbf{1 , 2 5 3 , 9 0 9}$ in 2020 with population conversion factor Region cascade from this 474012 population under PHS (Table 3).

Table 3: Number of HFs in the assessed Woreda of Awi zone, Amhara Region, June 15-25/2020 \begin{tabular}{|l|l|l|l|}
\hline S.No & Name of Woreda & Total Population & Number of HFs \\
\hline
\end{tabular} 


\begin{tabular}{|l|l|l|l|l|l|}
\hline & & & HC & HP & Hospital \\
\hline 1 & Chagni & 48,260 & 2 & 2 & 0 \\
\hline 2 & Ankasha Guagusa & 108,376 & 2 & 2 & 0 \\
\hline 3 & Banja & 99,967 & 2 & 2 & 0 \\
\hline 4 & Zigem & 109,255 & 2 & 2 & 0 \\
\hline 5 & Guagusa Shekudad & 108,154 & 2 & 2 & 0 \\
\hline & Total & $\mathbf{4 7 4 , 0 1 2}$ & $\mathbf{1 0}$ & $\mathbf{1 0}$ & $\mathbf{0}$ \\
\hline
\end{tabular}

\section{Current Malaria test results}

PHEM reports of 2019/2020 showed that1, 43131 total malaria fever cases were examined by RDT or Microscopy regionally. Of which 43131 confirmed malaria cases were reported in the Awi zone. Of this 33666 (78\%) and 9465 (22\%) were Plasmodium falciparum and Plasmodium Vivax respectively. Malaria accounts for $16.6 \%$ of the total outpatient cases and $0.07 \%$ of the inpatient cases. Of the total malaria cases, 6255 (14.5\%) were under-five children, $21 \%$ were people aged from 5-14 and the rest 64.5\% (27843) were above 15 years old. However, there were $1(0.02 \%)$ deaths due to malaria and pregnant mother were $596(1.5 \%)$ (Fig. 5).

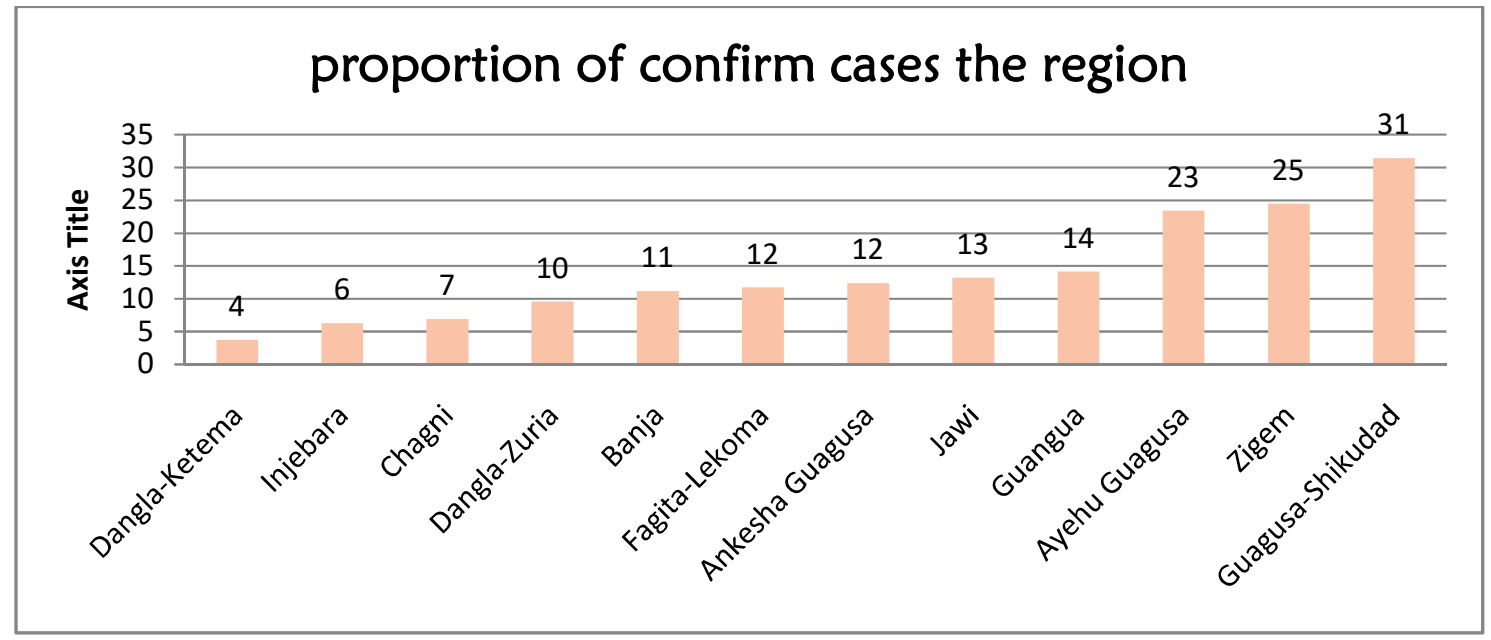

Figure 5: Proportion of Confirmed Malaria Cases by Reporting Zone of Awi Zone Amhara Region, Ethiopia 2020.

During the same year, Awi Zone also reported 259,009 (35.6\% of the region) total malaria cases examined by RDT or Microscopy, of which 18,726 (58\%) from the zone are confirmed (Fig. 6). 


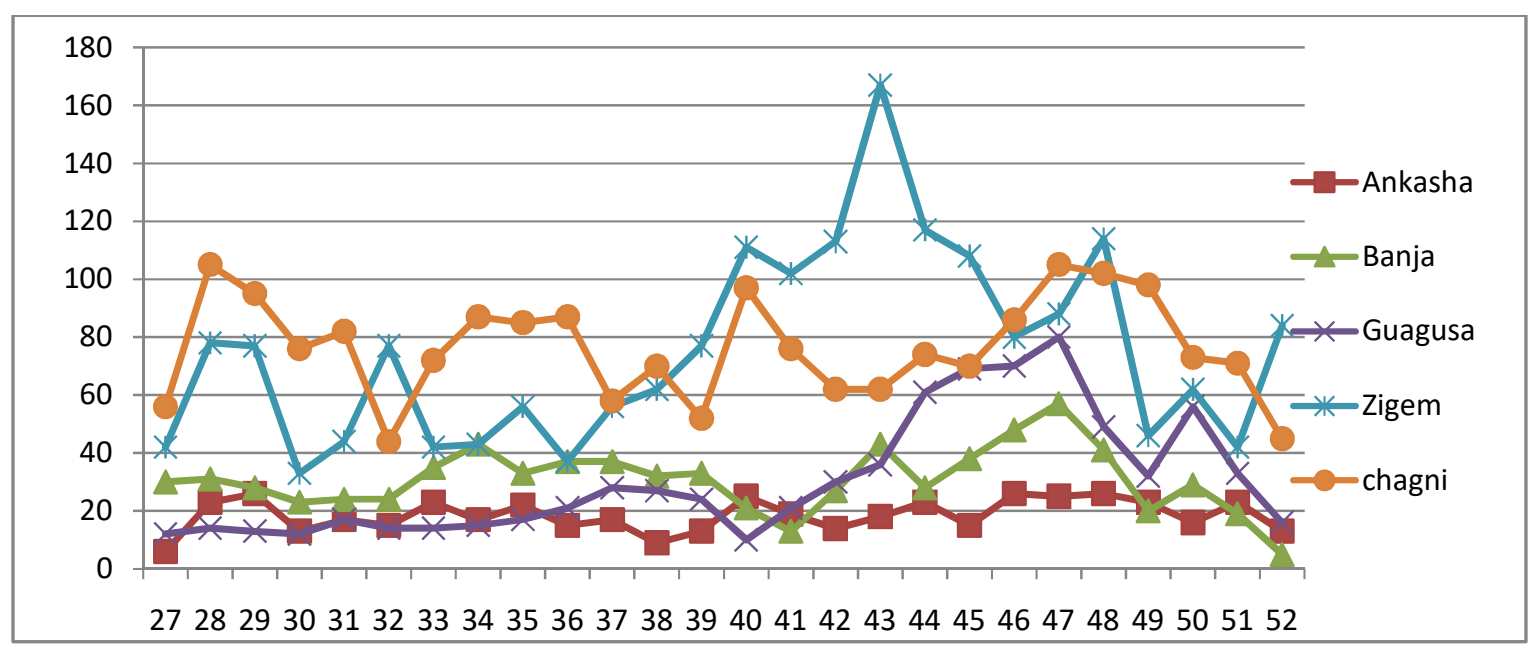

Figure 6: Trends of Confirmed malaria cases by woreda and WHO Epidemiologic week of Awi Zone, woreda, Amhara region Ethiopia, 2020.

A Zone reported 43131 confirmed malaria cases were reported from July 2019 to August 2020. A total of 41959 and 8793 confirmed malaria cases were reported from the woredas Ankesha, Banja, Changi, Guasgusa, and Zigem respectively to Awi Zone from July 2019 to January 2020. Of these, 6430 (73\%) cases were positive for Plasmodium falciparum

The 2020 PHEM report also showed 8793 confirmed malaria cases were reported from 27 weeks up to WHO week 52. From these 6430 (73\%) and 2363 (27\%) were plasmodium falciparum and plasmodium Vivax respectively (Fig. 7).

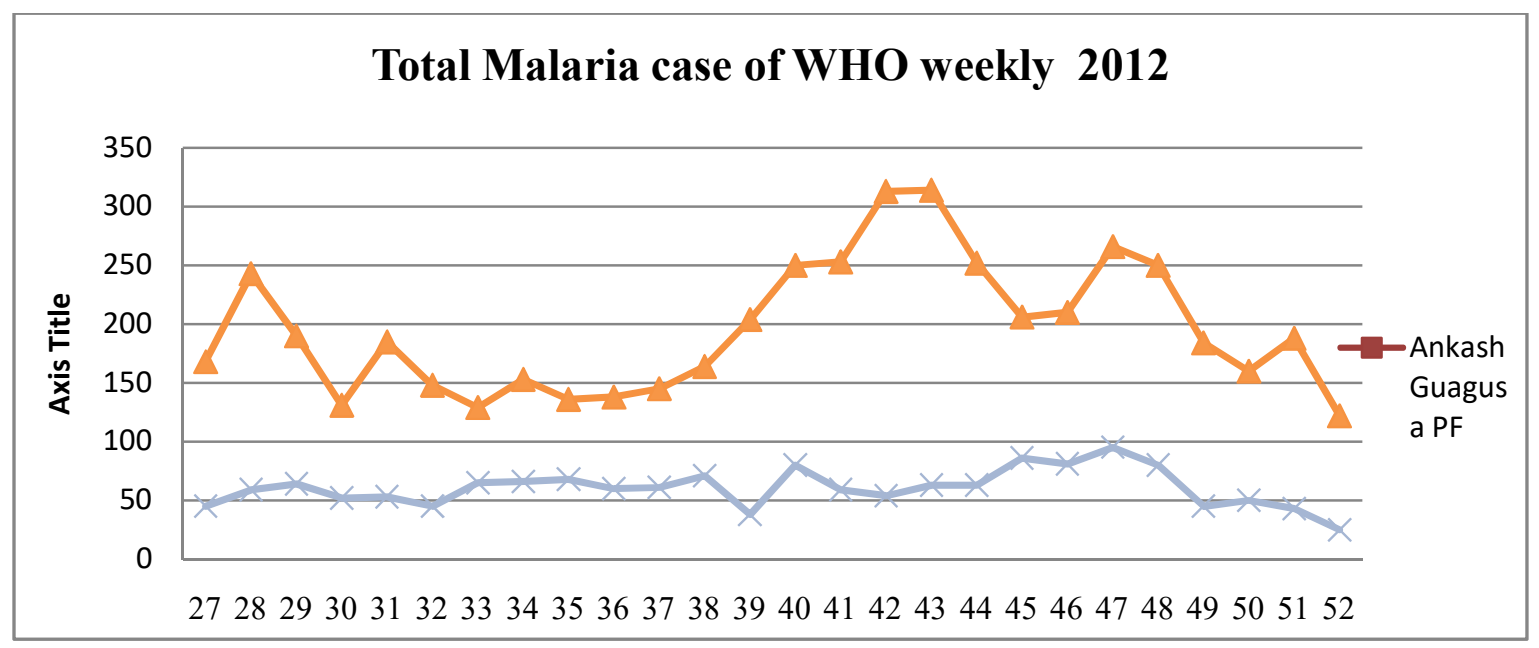

Figure 7: Trends of Confirmed malaria cases by species and WHO Epidemiologic week of Awi Zone, woreda, Amhara region Ethiopia, 2020. 


\section{DISCUSSION}

This study intended to the purpose of the evaluation of the trend of malaria in Awi zone, Amhara Region, Ethiopia, 2020 by seeing the effect of ivermectin on malaria reduction and by describing and measuring the core activity and supportive function of the surveillance system and surveillance attributes in the district, overall performance and using indicators to identify gaps or areas that could be strengthened.

We identified total Malaria cases of 259,009 outpatients, 43131 in-patients, and 1 death was recorded from 2011-2020 in Awi Zone. The most affected woredas in 2019 are Guasgusa Shekudad, Changi, and Zigem 314, 281, and 245 per 1000 respectively. Of all woredas in the Zone, Guasgusa Shekudad, Changi, and Zigem woredas are the most affected woredas from 2015-2020 (2096 per 10000 population). Of all Guasgusa-Shekudad woreda, is the most frequently affected woreda in the Zone from 2015-2019.

The analysis result showed that malaria occurred throughout the year, regardless of the woreda in the zone with annual malaria total prevalence rate for the calendar year 2015, it starts raising and was reaching its low point in 2019 which accounts 20 times lower than that of 2015. In addition to this, the confirmed malaria prevalence rate in this zone similarly declined from $340 / 1000$ in 2015 to $160 / 1000$ population in 2018 but radically decreased to $140 / 1000$ population in 2019.

Plasmodium specious varies from woreda to woreda. Distribution of Plasmodium ( ratio of plasmodium Falciparum to Plasmodium vivax) in Ankesha Guagusa, Banja, Guagusa Shekudad, Changi, and Zigem were 75:25,51:49, 88:12,72:28 and 86:14 respectively, and the total zonal ratio is $76: 24$ which is relatively matching with the national standard of the Plasmodium distribution of falciparum versus vivax, which is $65: 34(4,10)$.

However, in some woredas, the distribution of Plasmodium vivax species is higher than that of the spreading of falciparum which is not matched to the national guideline standards. These woredas include Guagusa Shekudad and Zigem. Some studies also supported these findings, which dedicated an increased proportion of Vivax malaria is consistent with a study at high altitude in the Butajira area (24), the decrease in Vivax malaria in highland-fringe areas could be explained by the high transmissibility of P.vivax. The P. falciparum prevalence increased by $75 \%$ in 2015 to $86 \%$ in $201 / 2015$, this was higher than the national report $2019(4,11)$.

This study provided that the following woredas, for example, Ankesha Guagusa, Banja, Guagusa Shikudad, Changi, and Zigem had the mean yearly anticipated occurrence of definite malaria higher than twenty per thousand human, this may be due to the higher number of

10 | P a g e 
reporting sites, arid areas and the presence of agricultural programs could affecting diseases spread where incidence be higher than expected and the rest of woreda be constantly under 5 reported malaria per thousand population per year $(4,12)$. In general, the utilize of prevalence while contrasting between woreda get better the capability of the zone to map property properly, develop targeted diseases organize labor, and permit an improved assessment of the program.

Supportive supervision helps to strengthen the capacity of staff and make sure that the proper skills are used appropriately; the mandatory logistics are in place, which planned activities are implemented in keeping with the schedule. Supervision is a vital support function that ensures success within the implementation of a surveillance system. A well-functioning system is often insured by regular and purposeful supervisory support. Within the woreda we visited during data collection, there have been not doing regular feedback and supportive supervision given to the lower levels. Supportive supervision was done just one time during the year 2019/20 which was but the expected plan (12).

Epidemic preparedness refers to the prevailing level of preparedness for potential epidemics and includes the availability of preparedness plans, storing, a designation of isolation facilities, setting aside of resources for outbreak response, at the least bit levels; There have been no wellorganized epidemic preparedness and response planning, and no financial and/ or support. This could cause weak case detection and response during epidemics.

Preparedness aims to strengthen capacity in recognizing and responding to public health emergencies through conducting regular risk identification and analysis, establishing partnerships and relationships, improving community participation, and implementing community-based interventions and strategic communication during the pre-emergency phase and ensuring their monitoring and evaluation (13).

The ability of the system to capture true diseases regarding malaria was assessed in this evaluation. On this basis, the predictive value positive for malaria by case definition was low in malarious districts of the Awi zone as the majority of them were greater than $35 \%$ for the year 2019/20. This was greater than the guideline developed by the Minister of Health of Ethiopia (4, 12).

The wholeness of coverage of malaria pointers for the PHEM scheme in the Awi zone within 2015 to 2019 , and accomplished that more than $96 \%$ had adequate excellence to produce measures of malaria occurrence by reporting woredas (14), Which is above the $80 \%$ WHO target $(13,14)$, viewing that everyone visited HFs were reporting to their respective level as per the quality within the country principle.

11 | P a g e 


\section{5: CONCLUSIONS}

The current PHS study revealed that the malaria incidence rate showed a remarkable decline. However, the annual parasitic incidence rate remains constant. This indicates that ivermectin did not affect malaria elimination.

Health care providers and health extension workers have a good understanding of the case definitions of malaria, other priority diseases, and surveillance. Report completeness and timeliness were good which above the national target both for the woreda and HFs. Laboratory diagnosis of malaria was available at all levels of the district and HFs were satisfactory. They only prepared a plan, there is no ready budget and resources to respond to any PHEM. Data analysis is only done at the zonal level, there was a lack of skill and resources for data analysis at the district and facility level. Outbreak investigation and response activities were done in a good manner. Supervision activities were poor at the zonal and district level, there were no program-specific supervision activities other than integrated supportive supervision which were conducted in each quarter. This could be due to the bad direction of all parties, insufficient helpful supervision and feedback, little or no law enforcement to the PHS activities, lack of incentives, appropriate training, sense of ownership, and logistics.

The finding suggests the importance of PHS training for focal persons and strict follow-up of the implementation of the acceptable surveillance system might improve PHEM capacity. Besides, minimizing the irrational case definition of diseases could also help to improve PHEM capacity.

\section{Declarations}

Ethics approval and consent to participate: Ethical clearance was obtained from the Institutional Review Board of the Jimma University and an official letter was submitted to the concerned bodies. The concerned bodies were informed to get the assurance of the study and confidentiality was maintained at all levels of the study. Informed consent was obtained from all participants and the Institutional Review Board of the Jimma University approved it with Ethical approval of Research protocol letter with its reference number IRB00010/2020.

\section{Informed consent}

Dear Sir/madam;

My name is and I am from I am conducting data collection on the

Trends of Malaria on Hotspot and Ivermectin Mass-drug Administration Zone of Amhara Regional State, 2020. The study being conducted by Mr. Chalachew Yenew from Jimma University. 
You are kindly requested to be included in the study, which has great importance in improving health. The interview will take a maximum of minutes.

No information concerning you as an individual will be passed to another individual or institution. Your participation will be based on your willingness and you have the right not to participate fully or partially. If you agree to be included in the study, I will start my question by asking general identification questions.

1. Agree to participate 2. Do not Agree to participate

Thank you for your cooperation!!!

Name of data collector Date Signature

Name of the supervisor Date Signature

Consent to Publish: Not applicable

Availability of data and materials: All data generated and analyzed during this study are included in this manuscript itself.

Competing interests: The authors have announced that they have no competing interests in nonfinancial includes political, personal, religious, ideological, academic, and intellectual and Financial issues.

\section{Funding: Not Applicable}

\section{Authors' contribution}

SM is actively involved during the conception of research issues and development of research proposals and CY has been writing of various parts of the research report and prepares the final manuscript. All authors read and approved the final manuscript.

\section{Acknowledgments}

Firstly, we would like to give our heartfelt thanks to the almighty God for giving us the knowledge and the support we need to do this study.

We also would like to acknowledge the Jimma and Debre Tabor University, College of Health Sciences for the arrangement and administrative support of our study.

\section{Abbreviations and Acronyms}

EFY: Ethiopian Fiscal Year, PHS: Public Health Surveillance, PH: Public Health, HO: Health Office, HC: Health Center, PV: Predictive Value, HP: Health Post, PHEM: Public Health Emergency Management. 


\section{References}

1. World Health Organization I 978-92-4-156572-1. World malaria report 2019. 2019.

2. The Carter Center, Atlanta, Georgia Held on March 8, 2013. 4th ANNUAL MALARIA CONTROL PROGRAM REVIEW Ethiopia and Nigeria Held on March 8, 2013, at The Carter Center, Atlanta, Georgia TABLE OF CONTENTS. (October 2013).

3. World Health Organization 2018. Malaria surveillance, monitoring \& evaluation: a reference manual,. 2018.

4. Ababa A. Ethiopia, malaria elimination strategic plan: 2021-2025. 2021;(August 2020):2021-5.

5. Shen M, Peng Z, Guo Y, Rong L, Li Y, Xiao Y, et al. International Journal of Infectious Diseases Assessing the effects of metropolitan-wide quarantine on the spread of COVID19 in public space and households. Int J Infect Dis. 2020;(2019):2019-21.

6. Chaccour C, Hammann F, Rabinovich NR. Ivermectin to reduce malaria transmission I. Pharmacokinetic and pharmacodynamic considerations regarding efficacy and safety. Malar J. 2017;1-16.

7. Centers for Disease Control (CDC). Guidelines for evaluating surveillance systems. MMWR Suppl. 1988;37(5):1-18.

8. World Health Organization, 20 Avenue Appia, 1211 Geneva 27 S (tel. . +41 22791 3264; fax: +41 22791 4857; e-mail: bookorders@who. int. Disease surveillance for malaria control:An operational manual. 2012;

9. Joseph A, Patrick N, Lawrence N, Lilian O, Olufemi A. Evaluation of Malaria Surveillance System in Ebonyi. 2017;101-4.

10. Tesfaye S, Belyhun Y, Teklu T, Medhin G, Mengesha T, Petros B. Malaria pattern observed in the highland fringe of Butajira, Southern Ethiopia : a ten-year retrospective analysis from parasitological and metrological data. 2012;3(5):1-8.

11. Delil RK, Dileba TK, Habtu YA. The magnitude of Malaria and Factors among Febrile Cases in Low Transmission Areas of Hadiya Zone, Ethiopia: A Facility Based CrossSectional Study. 2016;76:1-17.

12. Hill Z, Dumbaugh M, Benton L, Källander K, Strachan D, ten Asbroek A, et al. Training for mid-level managers (MLM ) 4. Supportive supervision. Glob Health Action. 2008;7(Mlm):1-34; Module 4.

13. Institute EH and NR, Centre PHEM, 2012, FebruaryBaba A, Ethiopia, Management public HE, Ethiopia G for, et al. Public Health Emergency Management. 2012;

14. Alemu T, Gutema H, Legesse S, Nigussie T, Yenew Y, Gashe K. Evaluation of public health surveillance system performance in Dangila district, Northwest Ethiopia: a concurrent embedded mixed quantitative/qualitative facility-based cross-sectional study. 2019;1-9.

15. $\mathrm{WHO} / \mathrm{AF} / \mathrm{WHE} / \mathrm{CPI} / 04,2019 \mathrm{WRO}$ for $\mathrm{A}$. This booklet comprises the following section of the Integrated Disease Surveillance and Response Technical Guidelines : Section 10 : Tailoring Integrated Disease Surveillance and Response (IDSR ) to Emergency or Fragile Health System contexts. 


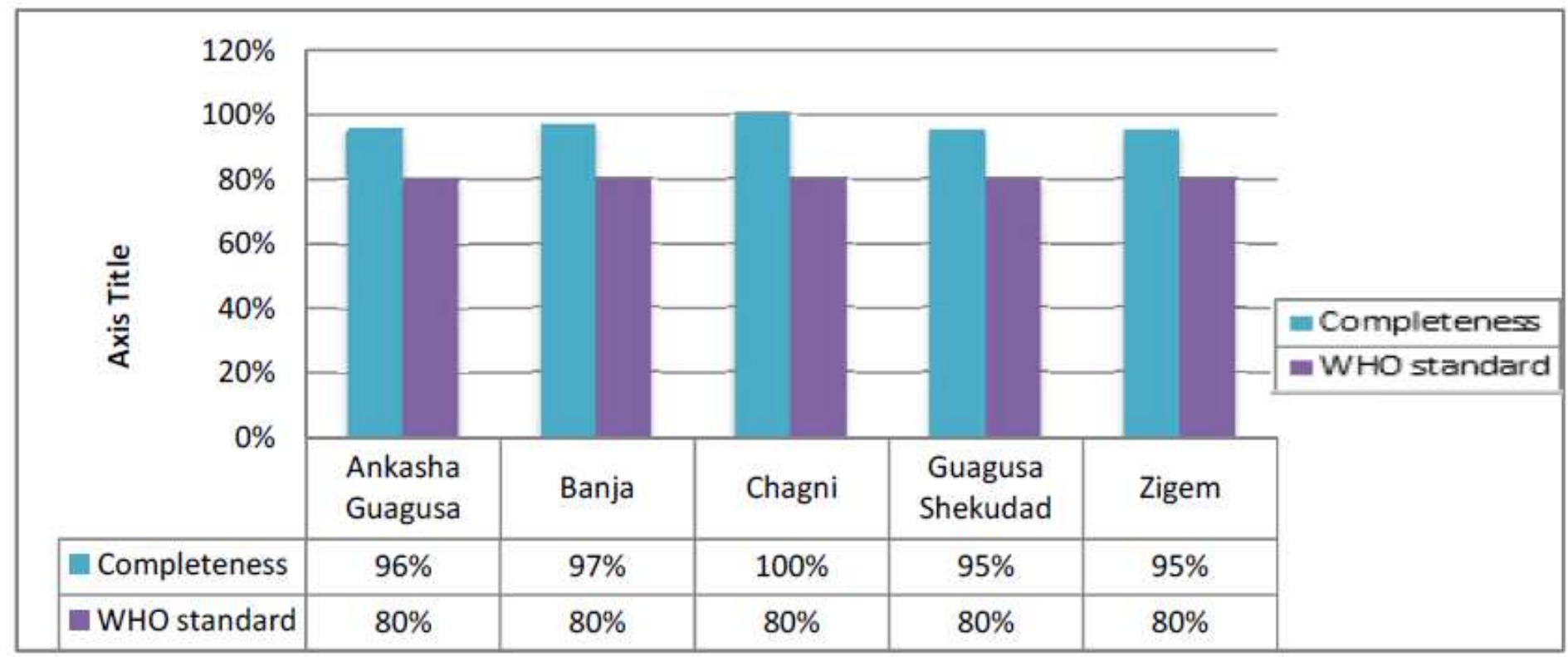

Figure 1

Timelines \& completeness of Governmental Health Facility Regional State, Ethiopia, 2020.

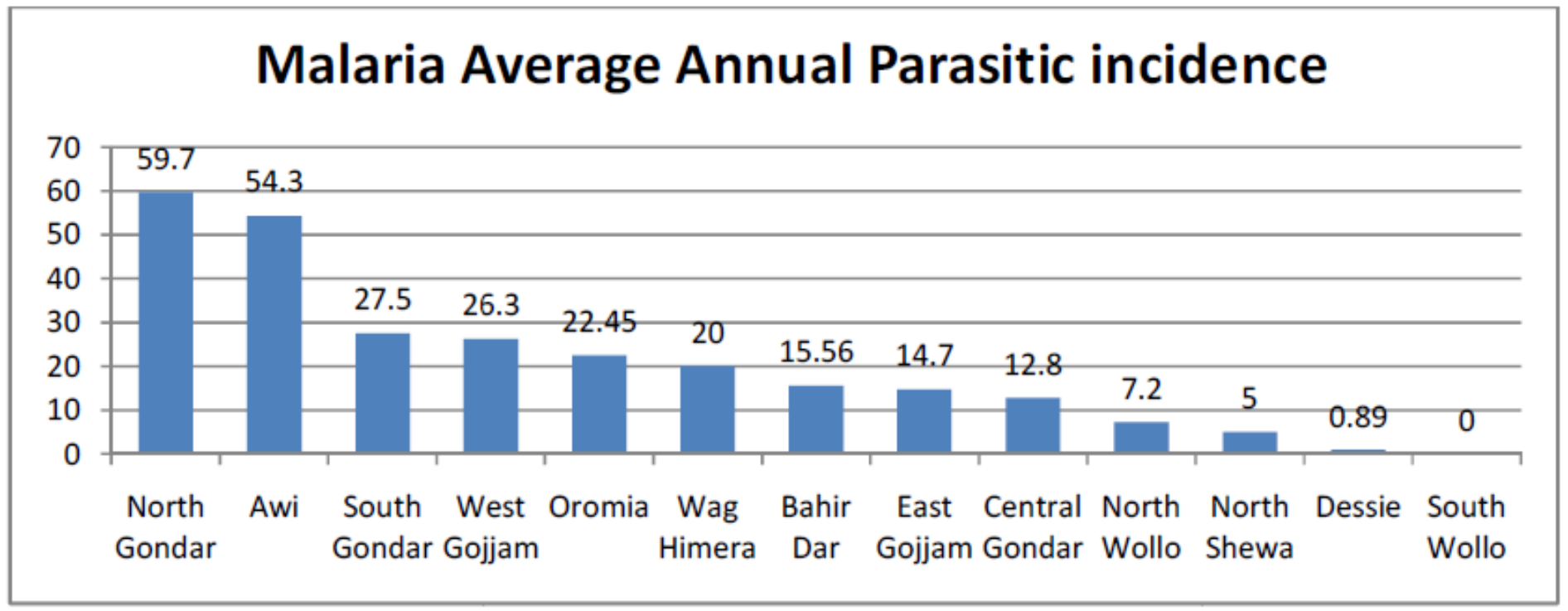

Figure 2

Malaria average annual parasitic incidence rate per 1000 population by zones in Amhara Region, Ethiopia, 2015-2019. 


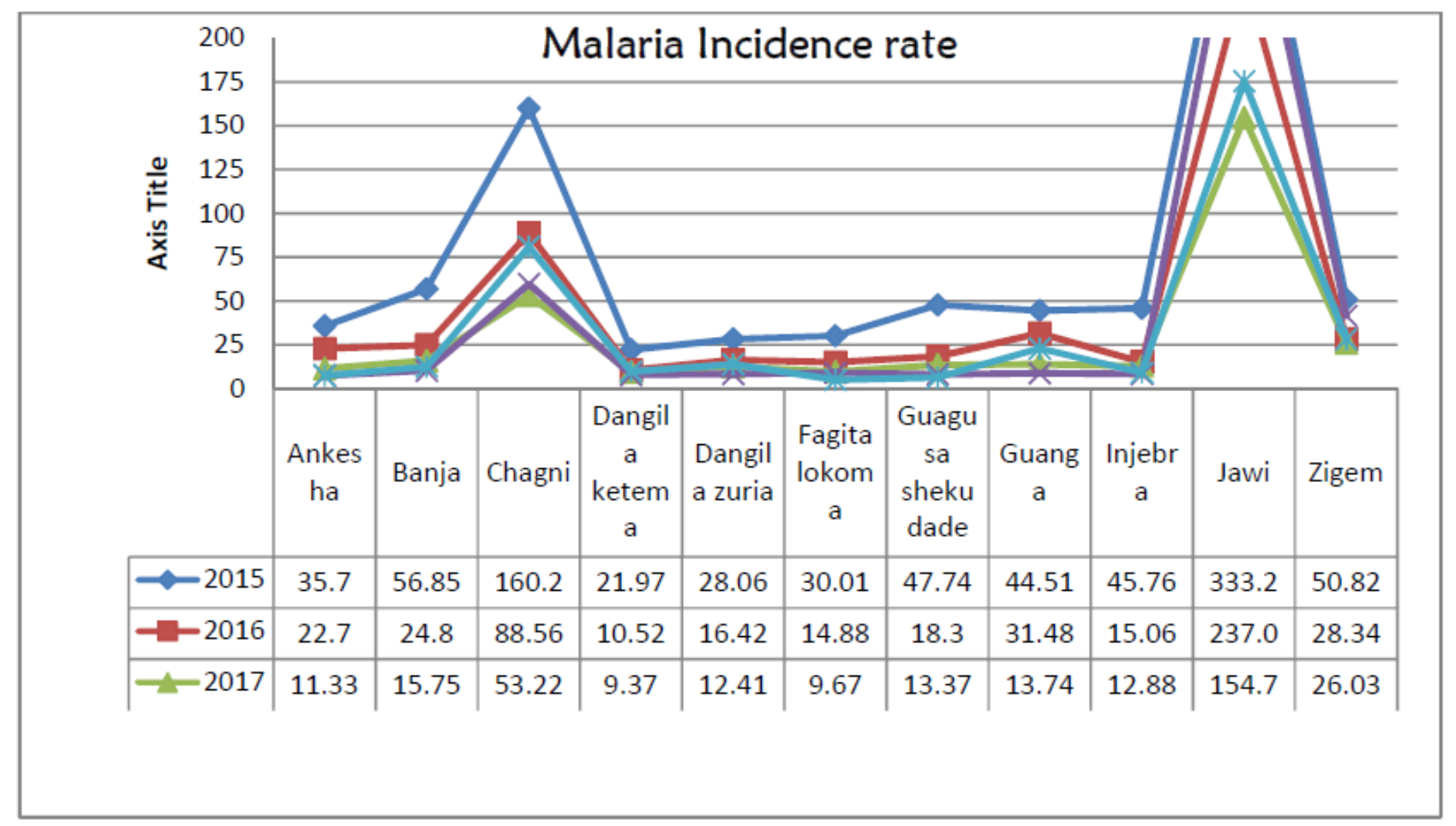

Figure 3

Trends of Malaria Incidence rate by Years of Awi Zone Amhara Region, 2020.

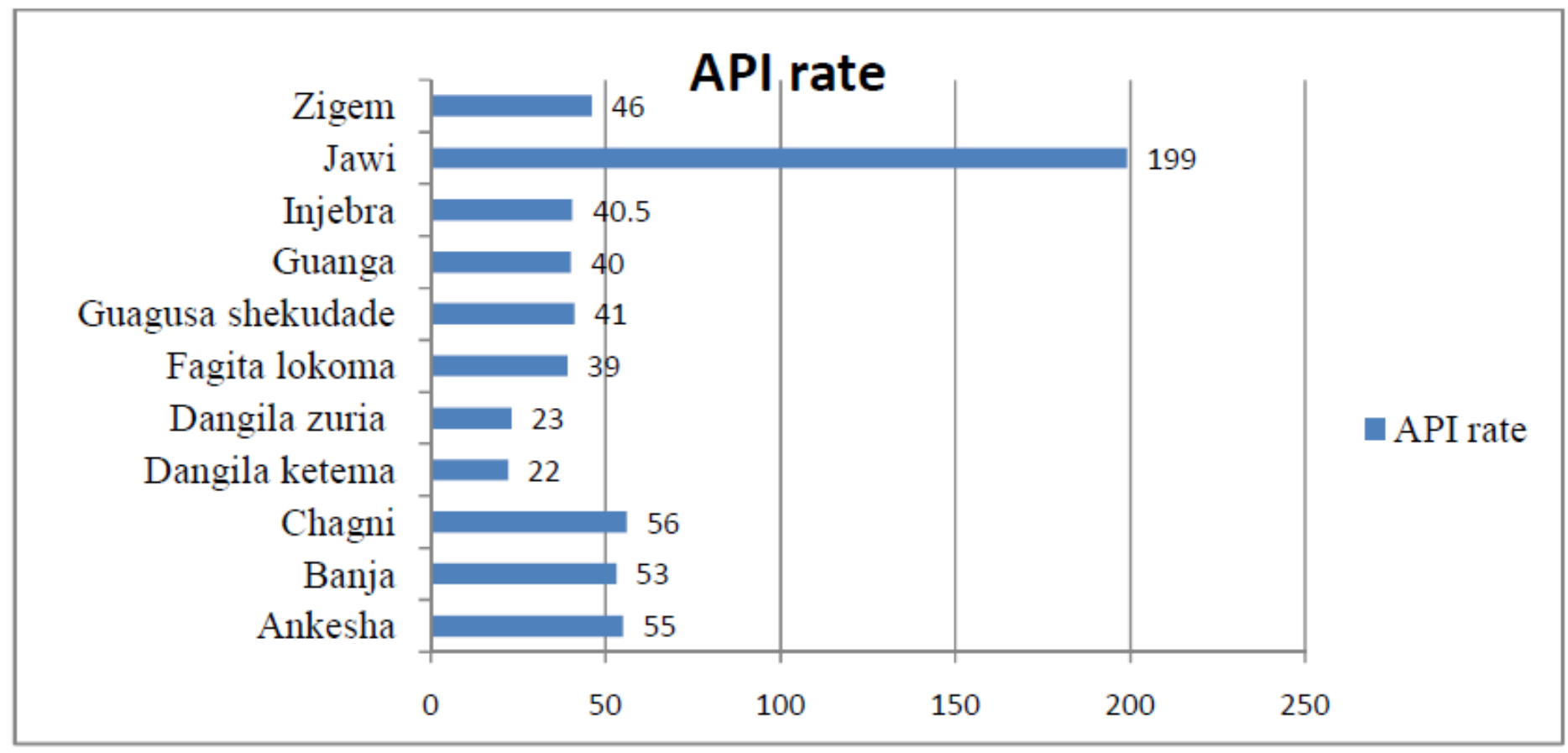

Figure 4

API rate of the study area, Awi Zone, Amhara Region, 2019. 


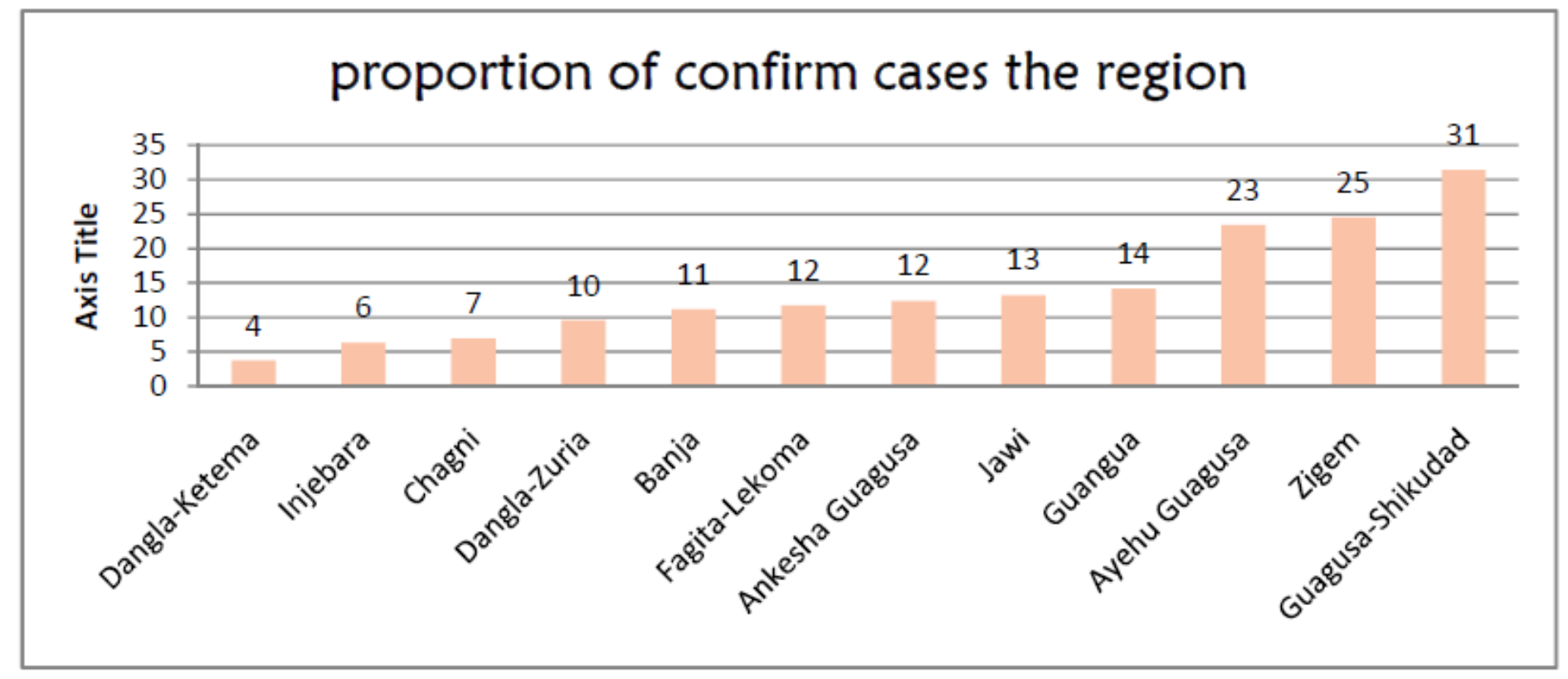

\section{Figure 5}

Proportion of Confirmed Malaria Cases by Reporting Zone of Awi Zone Amhara Region, Ethiopia 2020.

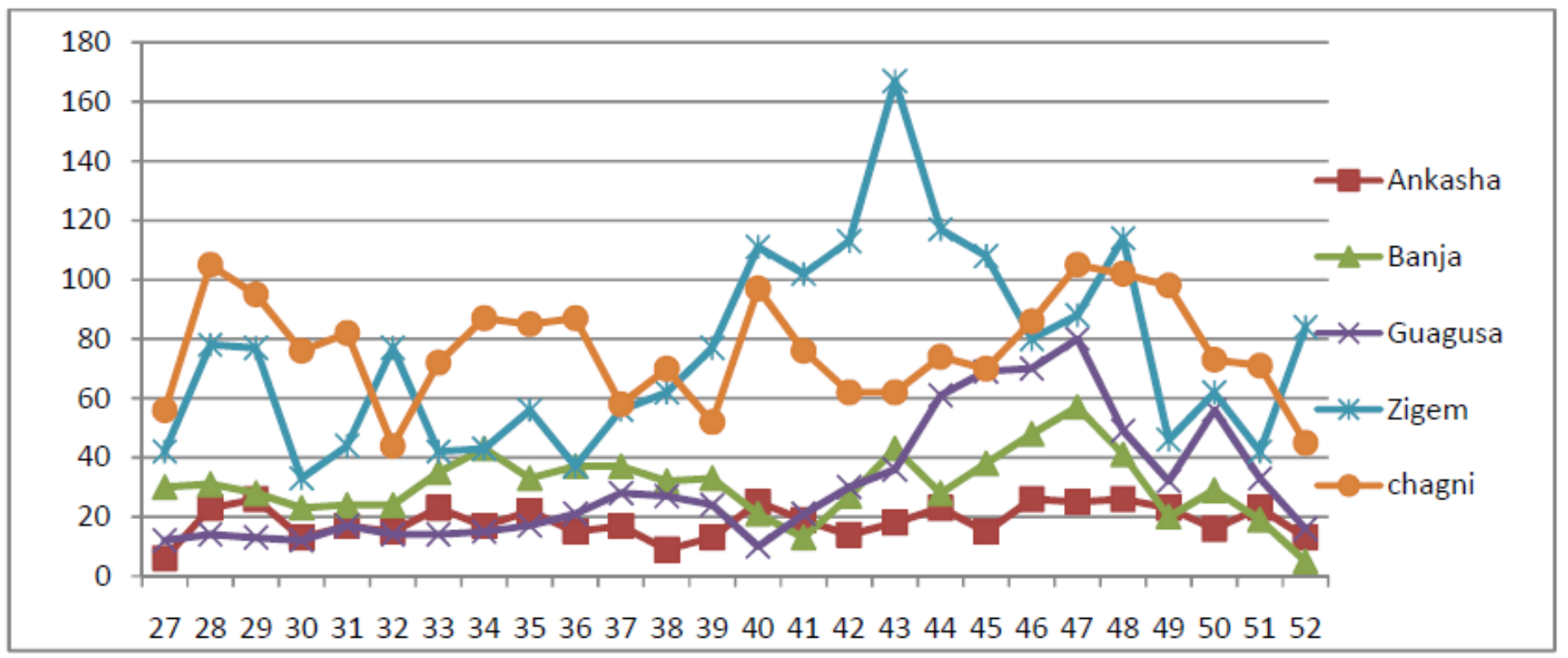

\section{Figure 6}

Trends of Confirmed malaria cases by woreda and WHO Epidemiologic week of Awi Zone, woreda, Amhara region Ethiopia, 2020. 


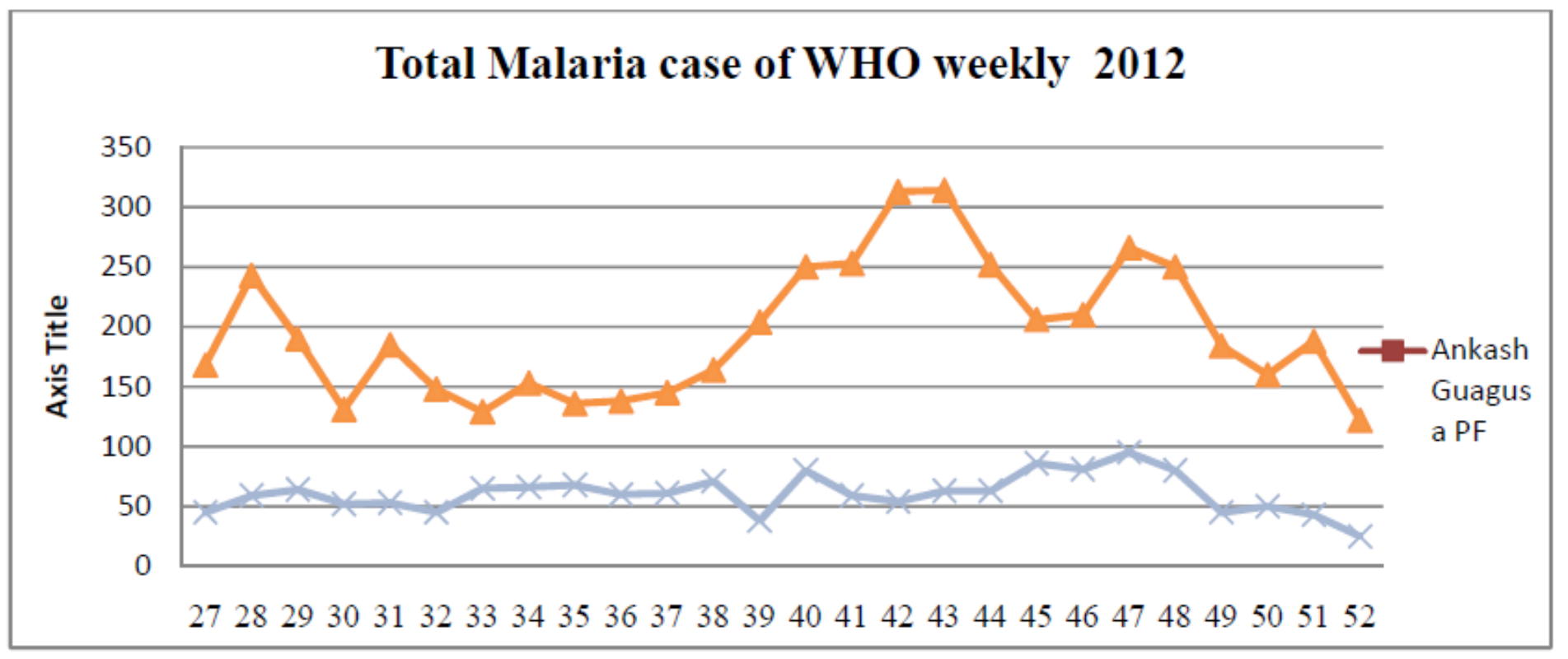

Figure 7

Trends of Confirmed malaria cases by woreda and WHO Epidemiologic week of Awi Zone, woreda, Amhara region Ethiopia, 2020. 